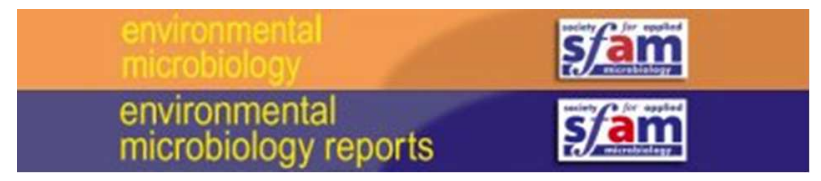

\title{
Growth phase-dependent control of R27 conjugation is mediated by the interplay between the plasmid-encoded regulatory circuit TrhR/TrhY-HtdA and the cAMP regulon.
}

\begin{tabular}{|r|l|}
\hline Journal: & Environmental Microbiology and Environmental Microbiology Reports \\
\hline Manuscript ID & EMI-2016-1487 \\
\hline Manuscript Type: & EMI - Research article \\
\hline Dournal: & Environmental Microbiology \\
\hline Complete List of Authors: & $\begin{array}{l}\text { Gibert, Marta; Universitat de Barcelona, Departament de Microbiologia } \\
\text { Paytubi, Sonia; University of Barcelona, Microbiology } \\
\text { Beltrán, Sergi; Gentre Nacional d'Anàlisi Genòmica (CNAG) } \\
\text { Juarez, Antonio; Universitat de Barcelona, Departament de Microbiologia; } \\
\text { Institut de Bioenginyeria de Catalunya (IBEC) } \\
\text { Balsalobre, Carlos; Universitat de Barcelona, Departament de Microbiologia } \\
\text { Madrid, Cristina; Universitat de Barcelona, Departament de Microbiologia }\end{array}$ \\
\hline Keywords: & $\begin{array}{l}\text { plasmid conjugation, R27, growth phase dependent control, cAMP, } \\
\text { transcriptional regulation, TrhR/TrhY-HtdA }\end{array}$ \\
\hline &
\end{tabular}

\section{SCHOLARONE ${ }^{m}$ \\ Manuscripts}


1 Growth phase-dependent control of R27 conjugation is mediated by the 2 interplay between the plasmid-encoded regulatory circuit TrhR/TrhY-HtdA 3 and the cAMP regulon.

4

$5 \quad$ Marta Gibert ${ }^{1 \#}$, Sonia Paytubi ${ }^{1 \#}$, Sergi Beltrán ${ }^{2}$, Antonio Juárez ${ }^{1,3}$, Carlos

6 Balsalobre $^{1 *}$ and Cristina Madrid ${ }^{1 *}$.

7 'Departament de Microbiologia, Universitat de Barcelona, Avda. Diagonal 643, 808028 Barcelona (Spain), ${ }^{2}$ Centre Nacional d'Anàlisi Genòmica (CNAG), Parc

9 Científic de Barcelona, Baldiri Reixac 4, 08028 Barcelona, (Spain), ${ }^{3}$ Institut de

10 Bioenginyeria de Catalunya (IBEC), Parc Científic de Barcelona, Baldiri Reixac

11 10, 08028 Barcelona (Spain).

\# Both authors contributed equally to this work.

${ }^{*}$ Corresponding authors: Carlos Balsalobre and Cristina Madrid.

15 Departament de Microbiologia, Universitat de Barcelona. Avda. Diagonal 643,

1608028 Barcelona, Spain.

17 Phone number: +34 934034622 (Carlos Balsalobre) / +34 934039382 (Cristina

Madrid). Fax: +34934034629.

E-mail addresses: cbalsalobre@ub.edu (Carlos Balsalobre), cmadrid@ub.edu

20 (Cristina Madrid).

22 Running title: Growth phase-dependent control of R27 conjugation.

23 Key words: plasmid conjugation, R27, growth phase dependent control, cAMP, transcriptional regulation, TrhR/TrhY-HtdA. 


\section{Originality-Significance Statement}

27 Plasmid conjugation is involved in the spreading of antibiotic resistance among

28 bacteria. Conjugation of IncHI1 plasmids, which are vectors of drug resistance

29 present in many pathogenic Gram-negative bacteria, occurs during bacterial

30 transit outside their warm-blooded hosts. In this report, we explored the

31 mechanisms underlying the regulation of plasmid conjugation by physiological

32 conditions. Our data indicated that the metabolic sensor cAMP plays a key role

33 in the growth phase-dependent control of IncHI1 plasmids transfer in

34 Escherichia coli via the TrhR/TrhY-HtdA plasmid conjugation regulators. This

35 work highlights the importance of regulatory crosstalk between conjugative

36 plasmids and the chromosome of recipient bacteria in environmental- and

37 physiological-mediated control of plasmid conjugation. 


\section{Summary}

40

41

Plasmids of the incompatibility group HI1 (IncHI1) have been isolated from several Gram-negative pathogens and are associated with the spread of multidrug resistance. Their conjugation is tightly regulated and it is inhibited at temperatures higher than $30^{\circ} \mathrm{C}$, indicating that conjugation occurs outside warm-blooded hosts. Using R27, the prototype of IncHI1 plasmids, we report that plasmid transfer efficiency in E. coli strongly depends on the physiological state of the donor cells. Conjugation frequency is high when cells are actively growing, dropping sharply when cells enter the stationary phase of growth. Accordingly, our transcriptomic assays show significant downregulation of numerous R27 genes during the stationary phase, including several tra (transfer) genes. Growth phase-dependent regulation of tra genes transcription is independent of H-NS, a silencer of horizontal gene transfer, and ppGpp and RpoS, regulators of the stationary phase, but highly dependent on the plasmidencoded regulatory circuit TrhR/TrhY-HtdA. The metabolic sensor cAMP, whose synthesis is chromosomally encoded, is also involved in the growth phase regulation of R27 conjugation by modulating $h t d A$ expression. Our data suggest that the involvement of regulators encoded by both chromosome and plasmid are required for efficient physiological control of IncHI1 plasmid conjugation. 


\section{Introduction}

The exchange of genetic material among bacteria, also known as horizontal gene transfer (HGT), contributes significantly to bacterial evolution. HGT promotes adaptation of the recipient bacteria to new environments through the acquisition of genes encoding metabolic pathways, adhesins, antimicrobial resistance, toxins, etc. Among the mechanisms underlying HGT, plasmid conjugation has become key in the rapid dissemination of antibiotic resistance among bacteria (Bennett, 2008)(Smillie et al., 2010). The transfer of plasmids by conjugation is a complex process requiring the expression of a large number of genes and proteins, some of them in large quantities. Therefore, a significant amount of bacterial resources is devoted to conjugation once triggered.

Conjugation is very tightly regulated, only promoted under certain environmental and physiological conditions to avoid deleterious effects on the fitness of the cell (Frost and Koraimann, 2010).

The plasmids of the incompatibility group $\mathrm{HI} 1$ (IncHI1) have been associated with multidrug resistance in several Gram-negative pathogens (Holt et al., 2011). Studies on R27, the prototype IncHI1 plasmid, have shown that there are at least 35 genes, called tra (transfer) genes, clustered in two separated regions (Tra1 and Tra2) that are required for conjugation (Lawley et al., 2002) (Lawley et al., 2003). This genetic organisation is shared by all the IncHI1 plasmids. The Tra1 region contains the origin of transfer (oriT) and three polycistronic operons: $\mathrm{H}, \mathrm{F}$ and $\mathrm{R}$. The $\mathrm{H}$ operon encodes relaxosome components and the coupling protein TraG, the F operon encodes proteins needed for $\mathrm{H}$-pilus biosynthesis, and the $\mathrm{R}$ operon encodes regulatory proteins involved in the transcriptional activation of many tra genes (Lawley et al., 2002) (Gibert et al., 2014). The Tra2 
region contains the operons $A C, A N$ and $Z$. The $A C$ operon encodes structural proteins of the mating pair formation complex and H-pilus, including the pilin TrhA. The AN operon encodes proteins of the mating pair formation complex and the regulatory protein $\mathrm{HtdA}$, whilst the $\mathrm{Z}$ operon encodes an entry exclusion system that stops cells carrying an IncHI1 plasmid from undergoing redundant conjugation (Gunton et al., 2008). Two groups of partition genes involved in plasmid segregation are located upstream (parM and parR) and downstream (parA and parB) of the Z operon (Rooker et al., 1999) (Lawley et al., 2003). Regulation of IncHI1 plasmid conjugation has been studied previously using as a model the transfer of the R27 plasmid between Escherichia coli cells. Environmental factors such as osmolarity, anaerobiosis, quorum sensing and acidity do not significantly affect the frequency of R27 conjugation (Alonso et al., 2005). To date, the only environmental condition described as affecting IncHI1 plasmid conjugation is temperature. Remarkably, maximal conjugation frequencies are detected at low temperatures between $22^{\circ} \mathrm{C}$ and $30^{\circ} \mathrm{C}$, whereas at $37^{\circ} \mathrm{C}$ conjugation is barely detectable. This thermoregulation suggests that IncHI1 plasmids transfer is enhanced in water and soil environments (Taylor and Levine, 1980) (Maher and Taylor, 1993). In this report, we describe that efficiency of R27 plasmid transfer strongly depends on the physiological state of the donor cells. Conjugation frequency is high when cells are actively growing (in the logarithmic phase), but drops sharply when cells enter the stationary phase of growth, correlating with the significant downregulation of the transcriptional expression of several R27 genes, including many tra genes. The regulatory circuit formed by TrhR/TrhY and HtdA has been described to modulate the expression of four out of six tra operons (Gibert et al., 2014). 
110 Here, we show that this circuit plays a crucial role in the growth phase-

111 dependent control of R27 conjugation. Moreover, the metabolic sensor cAMP

112 participates in the growth phase regulation of R27 conjugation by modulating

$113 h t d A$ expression. Our results suggest that an interplay between chromosomal

114 and plasmid-encoded factors is required for efficient physiological control of

115 IncHI1 plasmids conjugation. 


\section{Results}

118 R27 transfer is promoted in actively growing cells.

119 The effect of the physiological state of the bacterial cells on IncHI1 plasmid

120 conjugation was studied using the R27 plasmid. Conjugation frequencies at the

121 permissive temperature $\left(25^{\circ} \mathrm{C}\right)$ were monitored using donor and recipient cells

122 cultures grown up to an $\mathrm{OD}_{600 \mathrm{~nm}}$ of 0.4 and 2.0 , from now denoted respectively

123 as cultures in logarithmic (log) and early stationary (e-stat) phase of growth (Fig.

$1241 \mathrm{~A})$. Conjugation frequency was affected by the physiological state of the donor

125 cells, since R27 plasmid was more efficiently transferred from actively growing

126 cells (log) than e-stat cells (Fig. 1B). The physiological state of the recipient

127 cells did not notably affect conjugation efficiency.

Most of the genes involved in R27 plasmid transfer are upregulated at the transcriptional level in log cells.

130 To elucidate the mechanism behind the observed differences in conjugation frequency, transcriptomic analyses were performed to compare the expression pattern of the R27-encoded genes in log and e-stat cells. RNA samples from AAG1(R27) cultures grown at $25^{\circ} \mathrm{C}$ to either the log or e-stat phase were ensure that the accepted ratio was not based on very low signal strengths, we only considered genes with a signal value higher than the arbitrary value of 100 units of fluorescence intensity in at least one of the conditions. The fold change 
141 in the transcriptional expression between log and e-stat phase cells is shown in

142 Figure 2A and Table S1. Of the 205 R27 genes, 68 showed growth phase-

143 dependent expression, representing $33 \%$ of the genes. A total of 42 genes had

144 higher expression in the log phase, whereas 26 genes were expressed more in

145 the e-stat phase. Among the genes with altered expression, 21 belonged to the

146 tra operons, representing over $60 \%$ of the tra genes. Expression of tra genes is

147 indicated in both relative and absolute values in Figure 2A (black bars) and 2B,

148 respectively. As a control, the mRNA level of 7 selected genes, representing all

149 six tra operons, was monitored by RT-PCR (Fig. 2C). The results obtained were

150 in agreement with the transcriptomic data.

151 Consistent with the increased conjugation frequency detected in log cells, 18 of

152 the 21 tra genes were induced in actively growing cells, including those encoding crucial proteins for conjugation such as the pilin TrhA and the relaxase Tral (see Table S1 for a functional description of the affected genes). By contrast, only three genes ( $h t d A, h t d F$ and $h t d K)$ were induced in e-stat cells. Interestingly, $h t d A$ encodes a repressor of the transcriptional expression of the H, F, Z and AC operons (Gibert et al., 2013).

When considering genes located outside the tra operons, the expression of 47 genes was significantly affected by the growth phase, with 24 being highly expressed in log cells and 23 in e-stat cells. Although very few non-tra R27 genes have been fully characterised, protein homology studies allowed us to predict the putative function of some of them (Table S1). Among the genes induced during the log phase, some encode proteins that might contribute to efficient conjugation, such as partition proteins (R0013, R0014, R0042 and R0153),, DNA helicase (R0003), muramidase (R0130) and proteins involved in 
166 the turnover of disulphide bonds (R0131 and R0135) (Guynet et al., 2011)

167 (Gruber et al., 2016)(Zahrl et al., 2005)(Elton et al., 2005). Among the 23 genes

168 more highly expressed in e-stat than log cells, we were only able to assign a

169 putative function to two of them, a transposase (R0148) and a citrate

170 transporter (R0144), which are not involved in plasmid conjugation.

171 The master regulators of the stationary phase, ppGpp and RpoS, and the

174 The significant repression of R27 conjugation when cells enter the stationary phase of growth is similar to that observed for cellular processes regulated by ppGpp. The concentration of this secondary messenger increases rapidly in the interphase between the log and e-stat phases, mediating the changes in gene expression, as well as affect a large set of genes by promoting, to different degrees, the activity of RpoS, the stationary-phase sigma subunit (Magnusson et al., 2005)(Battesti et al., 2011). To determine whether the growth phase control of R27 conjugation is mediated by ppGpp and/or RpoS, conjugation experiments were performed using donor cells lacking either ppGpp or RpoS (Fig. 3). In $\mathrm{ppGpp}^{0}$ and $r p o S$ derivative strains, although a slight increase was observed in the conjugation frequency in e-stat cells, there was still a pronounced difference in the conjugation frequency between log and e-stat cells. Therefore, ppGpp and RpoS did not seem to be essential for the growth phase dependency of R27 conjugation. However, they could still be involved in the fine tuning of R27-transfer control. 
190 It has been postulated that H-NS silences HGT by repressing the expression of

191 suddenly acquired DNA to diminish its potential deleterious effect on the fitness

192 of the cell (Doyle et al., 2007). Remarkably, a gene encoding an H-NS-like protein has been identified in several conjugative plasmids, including R27 (reviewed by Dorman, 2014). Previous studies have described the role of H-NS in repressing R27 tra gene expression (Forns et al., 2005) (Gibert et al., 2014).

We performed conjugation experiments using donor cells deficient in both chromosomal- and plasmid-encoded H-NS orthologues (Fig. 3). As expected, a higher conjugation frequency was detected in the H-NS deficient strain compared to WT in both the log and e-stat cells. However, there was still a significant difference in conjugation frequency being more than 1,500-fold higher in the log cells than in e-stat cells. These results indicated that H-NS is not involved in the growth phase control of R27 conjugation.

The TrhR/TrhY-HtdA regulatory system is involved in the growth phasedependent regulation of $\mathrm{R} 27$ conjugation.

The R27-encoded TrhR, TrhY and HtdA form a regulatory circuit that controls transcription of the tra operons and consequently modulates R27 conjugation (Gibert et al., 2013)(Gibert et al., 2014). TrhR and TrhY have an essential role establishing protein-protein interactions. Interestingly, $h t d A$ was one of the few genes induced in the e-stat phase (more than 3-fold compared to the log 
215 phase), while trhR was slightly induced in log cells (1.8-fold) (Fig. 2 and Table

216 S1). These fluctuations in expression levels, particularly in $h t d A$, may explain

217 the low R27 conjugation frequency observed in e-stat cultures.

218 To dissect the role of TrhR/TrhY and HtdA in growth phase-dependent control,

219 conjugation studies were performed with R27 variants carrying mutations for

220 either trhR or $h t d A$ (Fig. 3). In accordance with the requirement of TrhR/TrhY for

221 the expression of tra operons (Gibert et al., 2014), conjugation was completely

222 abolished in the trhR derivative in both log and e-stat donor cells. By contrast,

$223 h t d A$ mutation elicited derepression of R27 conjugation in both log and e-stat

224 donor cells. Strikingly, the difference in the averages of the conjugation

225 frequency between log and e-stat cells with WT R27 plasmid (2.78E-3 vs 4.43E-

226 7) was reduced more than 300 times in the absence of $h t d A(3.23 \mathrm{E}+0$ vs $1.59 \mathrm{E}-$

227 1). Consistent with the fact that $h t d A$ expression was induced in the e-stat

228 phase, a greater derepression of R27 conjugation was observed in the htdA

229 mutant strain in the e-stat phase compared to the log phase. These results

230 suggest that $\mathrm{HtdA}$ plays an important role in the repression of R27 conjugation

231 observed when cells enter the stationary phase. The transcriptional expression

232 of $\operatorname{trh} A$, encoding the $\mathrm{H}$ pilus major subunit, was studied by RT-PCR in log and

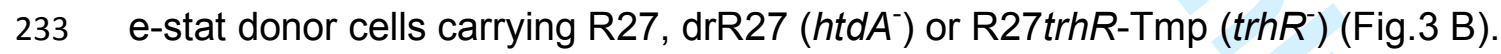

234 In line with the conjugation frequency data in log cells, a pronounced induction

235 of trhA transcription was observed in log cells carrying the R27 plasmid. The

236 growth phase control of $\operatorname{trh} A$ was abolished in both $h t d A$ and $\operatorname{trh} R$ derivative

237 strains since trhA expression was only slightly increased in log cells compared

238 to e-stat cells. As expected from the roles of HtdA and TrhR in tra gene

239 expression, $\operatorname{trh} A$ mRNA levels in the $h t d A$ mutant were higher than in cells 
carrying the WT plasmid, and lower in the trhR strain (note that the amount of

241 total RNA required for efficient $\operatorname{trh} A$ detection was $0.1,1$ and $10 \mathrm{ng}$ for $h t d A$, WT and trhR strains, respectively). Thus, these results were consistent with the observed differences in conjugation frequency (Fig. 3A).

244 Given that HtdA is involved in the growth phase-dependent control of R27 conjugation (Fig. 3A) and that $h t d A$ expression is significantly higher in the estat phase (3-fold, see Fig. 2 B), we postulate that fluctuations in the level of the antiactivator ( $\mathrm{HtdA})$ and, consequently, in the availability of functional activators (TrhR/TrhY), determine the frequency of R27 conjugation in log and e-stat donor cells. The effect of increasing the amounts of the different regulatory components on conjugation frequency was determined in both log and e-stat

\section{cAMP controls R27 conjugation in an HtdA-dependent manner.}

A relevant question to address is how fluctuations in $\mathrm{HtdA}$ expression occur in response to the physiological state of the cell. During a random mutagenesis experiment searching for regulators of the R27 tra operons, we found that mutation in the cya gene, encoding cAMP synthetase, affected expression of

262 the tra operons (data not shown). These findings indicated that cAMP may be 263 involved in regulating the synthesis of the conjugative apparatus. In 
enterobacteria, the secondary messenger cAMP acts as a physiological sensor.

cAMP, in a complex with cAMP receptor protein (CRP), can bind DNA and

regulate the expression of a vast number of genes (Shimada et al., 2011).

Although initially described as playing a role in regulating metabolic gene expression, CAMP-CRP is now known to modulate different cellular processes (Zheng et al., 2004). Furthermore, cAMP-CRP has been reported to be involved in the growth phase-dependent expression of colonisation factors in E. coli (Müller et al., 2009). Here, we studied the effect of cya mutation in the expression of the conjugative apparatus by RT-PCR analyses of the trhA transcript. cAMP deficiency sharply reduced trhA expression (Fig. 5A). To further characterise the involvement of cAMP in regulating R27 transfer, conjugation frequencies were measured for both cAMP-deficient and -proficient cells (Fig. 5B). In log cells, mutation in cya significantly decreased conjugation frequency (> 4,000-fold). Moreover, the effect of the cya mutation was blocked when the cells lacked HtdA (drR27), indicating that HtdA is crucial for cAMPmediated control of R27 conjugation. In e-stat cells, no significant differences were detected between the WT and cya mutant strains, suggesting that cAMP plays a key role in controlling conjugation in log cells. Since cAMP forms a complex with CRP, R27 conjugation was also monitored in a crp mutant strain (Fig. 5C). In log cells the frequency of conjugation drops in the crp strain and this reduction was lower in the absence of HtdA. Taken together, our results indicated that cAMP-CRP promotes R27 transfer, possibly by repressing HtdA expression. To explore this hypothesis, $h t d A$ transcriptional expression was further studied using a transcriptional fusion between the $h t d A$ promoter and lacZ inserted into the chromosomal attB locus. Along with the observed 
289 significant drop in R27 conjugation, the absence of either cAMP or CRP

290 increased $h t d A$ transcription (Fig. 5D). htdA expression in crp and cya mutants

291 was upregulated in both the presence and absence of the R27 plasmid,

292 suggesting that the repression effect was independent of the presence of any

293 R27-encoded factor. Further experiments were performed by adding exogenous

294 cAMP to cya mutant cultures (Fig. 5E). The htdA upregulation observed in the

295 cya mutant strain was suppressed after adding cAMP. Thus, our data indicate a

296 link between the cAMP-CRP regulon and the TrhR/TrhY-HtdA regulatory circuit

297 controlling R27 conjugation. 
300

301

302

IncHI1 plasmids - including R27 - are antibiotic resistance-spreading elements prevalent among pathogenic enterobacteria. Their transfer is repressed at temperatures above $30^{\circ} \mathrm{C}$, meaning that dissemination is likely to occur during bacterial transit in natural environments outside warm-blooded hosts (Maher and Taylor, 1993). Our data indicated that R27 conjugation, in addition to low temperature (permissive conditions), is promoted in actively growing cells. Transfer frequency was high when donor cells were in the exponential phase of growth and dropped during entry into stationary phase, after growth rate declined (Fig. 1A). The sensitivity of R27 transfer to the physiological state of the cell was restricted to the donor cell (Fig. 1B). Transcriptional profiling indicated that many R27 genes involved in plasmid transfer are induced in log cells (Fig. 2), suggesting the existence of a regulatory mechanism controlling the transcriptional expression of plasmid transfer genes in a growth phasedependent manner. Given that plasmid conjugation uses a large amount of energy and resources, our results suggest that when encountering metabolic stress, cells suppress IncHI plasmid conjugation to avoid compromising survival of the donor cell. In harsh conditions, these mechanisms minimise the deleterious effect of conjugative plasmids on bacterial fitness. This plasmid accommodation within the cells lets them act as successful vehicles for DNA spreading among bacteria. An example of coevolution between plasmids and their hosts has been shown with R27 and other IncHI1 plasmids (Paytubi et al., 2014). These plasmids have developed a complex interaction with the bacterial chromosome, resulting in an interplay that increases bacterial fitness at low temperatures, thus facilitating the survival of Salmonella outside its host. 
324 The impact of the growth phase, nutrients and energy availability on conjugation

325 has been studied with other plasmids. F plasmid transfer in E. coli has been

326 reported to be inhibited in the stationary phase by a mechanism involving $\mathrm{H}-\mathrm{NS}$

327 (Frost and Manchak, 1998) (Will et al., 2004). The expression of the main

328 regulators of the $\mathrm{F}$ tra operon, traM and traJ, is significantly derepressed in an

329 hns mutant, particularly when the cells enter the stationary phase (Will et al.,

330 2004). However, a different observation has been reported for the F-like

331 plasmid, pSLT, in Salmonella. pSLT conjugation does not decline in the

332 stationary phase and traJ expression is inhibited by H-NS in both the

333 logarithmic and stationary phases of growth (Camacho et al., 2005). Growth

334 rate and nutrient levels also affect IncP and Incl1 plasmids transfer efficiency,

335 although the regulatory mechanism remains unknown (Fox et al., 2008)(Händel

336 et al., 2015). Moreover, active growth is required for efficient plasmid transfer,

337 which may account for the limited plasmid spread detected in slow-growing cells

338 during biofilm formation (Merkey et al., 2011).

339 Our data demonstrated that the stationary phase regulators $p p G p p$ and RpoS

340 were not essential, but could affect the growth phase-dependent transfer of R27

341 to some extent (Fig. 3). Recently, we described that H-NS-mediated

342 thermoregulation of R27 conjugation occurs by repressing the expression of the

343 two plasmid-encoded activators, TrhR and TrhY, at high temperatures (Gibert et

344 al., 2014). In the present study, H-NS did not seem to be crucial in the growth

345 phase control of R27 conjugation since there was still a significant difference in

346 the conjugation frequency between log and e-stat cells in an H-NS-deficient

347 strain (Fig. 3). Interestingly, growth phase-dependent regulation was strongly

348 governed by the regulatory circuit TrhR/TrhY-HtdA, since it disappeared when 
349 the levels of the regulators were altered either by deletion or ectopic

350 overexpression (Figs. 3 and 4). Moreover, the expression profile of the

351 regulators, particularly $\mathrm{HtdA}$, was consistent with their active role in the growth

352 phase-dependent regulation of R27 conjugation (Fig. 2).

353 In this report we show that factors other than temperature are required to

354 alleviate the HtdA-mediated repression. At the permissive temperature,

355 expression of the conjugative apparatus occurred mostly in actively growing

356 cells. This regulation may be needed to avoid the unnecessary synthesis of the

357 R27 conjugative apparatus when cells are under suboptimal physiological

358 conditions.

359

360

361

We observed that the growth phase-dependent control of R27 conjugation was abolished in a cAMP-CRP-deficient strain. Furthermore, the conjugation frequency in log cells dropped to that seen in e-stat cells in both cya and crp mutant strains (Fig. 5B, Fig. 5C). The effect of both cya and crp mutations on conjugation was much more pronounced for the R27 (htdA $\left.A^{+}\right)$than $\operatorname{drR} 27\left(h t d A^{-}\right)$ plasmid, suggesting that the pivotal role of cAMP in controlling R27 conjugation requires HtdA. We propose a model (Fig. 6), where cAMP-CRP is involved in maintaining low levels of $\mathrm{HtdA}$ during the logarithmic phase of growth, thus promoting R27 conjugation. In the early stages of the stationary phase, HtdA expression increases and, by counteracting the activators TrhR/TrhY, suppresses the expression of most of the tra genes, consequently reducing conjugation frequency. Consistent with the proposed model, htdA expression was derepressed in both cya and crp mutant strains and decreased when external cAMP was added (Fig. 5). Although further studies are required to elucidate the molecular mechanism underlying cAMP-CRP-mediated repression 
374 of $h t d A$ expression, our results clearly indicate that it is crucial for the

375 physiological control of R27 transfer. Indeed, the role of the metabolic sensor

376 cAMP in the growth phase control of transcriptional expression has been

377 previously described. Studies with the F-like plasmid pRK100 showed that,

378 similar to its effect on R27 conjugation, cya mutation reduced conjugal transfer

379 and CAMP-CRP stimulated the expression of the central positive regulator TraJ,

380 which is growth phase-dependent (Starcic et al., 2003). Moreover, cAMP has

381 been described to be involved in the repression of type 1 fimbriae in $E$. coli

382 during the log phase of growth (Müller et al., 2009).

383 Here, we show that R27 plasmid transfer depends on the physiological state of

384 the donor cell and is regulated by $\mathrm{HtdA}$ expression under the control of the

385 metabolic sensor cAMP. Plasmid conjugation can be considered an altruistic act

386 from the bacterial perspective or a selfish behaviour from the point of view of

387 the plasmid. In the latter, the main aim of a conjugative plasmid is to spread and

388 safeguard its genes. For some plasmids, such as R27, biological success is

389 achieved by promoting their spread without deleterious effects on the host cells.

390 Therefore, transfer must occur when the physiological conditions of the host

391 cells are optimal to trigger conjugation. Our data demonstrate that cAMP acts

392 as a physiological sensor that modulates the plasmid-encoded regulatory circuit

393 TrhR/TrhY-HtdA, the main controller of R27 conjugation. 


\section{Experimental procedures}

396

397

398

399

400

401

402

403

404

405

406

407

408

409

410

411

412

413

414

415

416

417

418

\section{Bacterial strains, plasmids and growth conditions}

The E. coli strains and plasmids used are listed in Table 1. Oligonucleotides are listed in Table S2. Bacteria were grown in LB (10 g/l NaCl, $10 \mathrm{~g} / \mathrm{l}$ tryptone and 5 g/l yeast extract). For mating experiments, strains were grown in Penassay broth (1.5 g/l meat extract, $1.5 \mathrm{~g} / \mathrm{l}$ yeast extract, $5 \mathrm{~g} / \mathrm{l}$ peptone, $1 \mathrm{~g} / \mathrm{l}$ glucose, 3.5 $\mathrm{g} / \mathrm{l} \mathrm{NaCl}, 1.32 \mathrm{~g} / / \mathrm{KH}_{2} \mathrm{PO}_{4}$ and $\left.4.82 \mathrm{~g} / / \mathrm{K}_{2} \mathrm{HP}_{4} \cdot 3 \mathrm{H}_{2} \mathrm{O}\right)$. For selecting transconjugants, M9 minimal medium plates were used, with the following composition: M9 salts (Sambrook et al., 1989), 0.2\% (w/v) lactose, $10 \mu \mathrm{M}$ thiamine and $1.5 \%(\mathrm{w} / \mathrm{v})$ Bacto agar. When needed, antibiotics were added at the following concentrations: $15 \mu \mathrm{g} / \mathrm{ml}$ tetracycline (Tc), $20 \mu \mathrm{g} / \mathrm{ml}$ chloramphenicol (Cm), $25 \mu \mathrm{g} / \mathrm{ml}$ kanamycin $(\mathrm{Km}), 50 \mu \mathrm{g} / \mathrm{ml}$ ampicillin (Amp), and $10 \mu \mathrm{g} / \mathrm{ml}$ trimethoprim (Tmp). For monitoring the lac phenotype, LB agar plates were supplemented with $40 \mu \mathrm{g} / \mathrm{ml}$ X-gal and $0.5 \mathrm{mM}$ IPTG. When required, cAMP was added at a final concentration of $5 \mathrm{mM}$. Inducing experiments of pBAD derivative plasmids were performed as described previously (Gibert et al., 2014).

\section{Strain construction}

The AAG1 rpoS, AAG1 cya, AAG1-AN cya, AAG1 crp and AAG1-AN crp strains were obtained by P1 transduction using the strains JW5437 (rpoS::Km), AAG1 cya (cya::Tmp) from a random mutagenesis experiment and BRE2055 (crp::Tc). All genetic constructs were confirmed by DNA sequencing. 


\section{Mating experiments}

420 Mating experiments were performed following the standard protocol for R27

421 conjugation (Taylor and Levine, 1980), slightly modified. In all cases, AAG1 and

422 its derivatives were used as donor cells and MG1655 as recipient cells. Cultures

423 of donor and recipient strains were grown in Penassay broth at $25^{\circ} \mathrm{C}$ in shaking

424 conditions to either an $\mathrm{OD}_{600 \mathrm{~nm}}$ of 0.4 (log phase) or 2.0 (e-stat phase). When

425 log-phase cultures were used, cell suspensions were concentrated to the

$426 \mathrm{OD}_{600 \mathrm{~nm}}$ of the e-stat cultures. Otherwise stated, recipient cells were grown to e-

427 stat phase. Mating frequency was calculated as the number of transconjugants

428 per donor cell.

$429 \quad \beta$-galactosidase assays

$430 \quad \beta$-galactosidase assays were performed as described by Miller (Miller, 1992).

431 Data are given as the means of duplicate determinations in at least three

432 independent experiments, plotted with standard deviations.

433

434

435

\section{Total RNA isolation}

Total RNA was isolated from three independent cultures grown at $25^{\circ} \mathrm{C}$ under shaking to either log phase $\left(O D_{600 n m}\right.$ of 0.4$)$ or e-stat phase $\left(O D_{600 n m}\right.$ of 2.0$)$.

The RNA was purified as previously described (Gibert et al., 2014). For microarray experiments, the purified RNA was concentrated using an RNeasy MiniElute Clean-up kit (Qiagen). Purity and quality of the RNA were tested by Bioanalyzer 2100 (Agilent Technologies).

\section{Reverse transcriptase (RT)-PCR assays}

Semi-quantitative monitoring of the mRNA levels was performed using the Transcriptor One-Step RT-PCR kit (Roche), as previously described (Gibert et 
443 al., 2014). Primer pairs used are described in Table S2. The relative amount of 444 cDNA was determined using the ImageJ software (Schneider et al., 2012).

\section{Microarray analysis}

446 Transcriptomic analysis was performed on a custom-designed DNA microarray 447 engineered by NimbleGen, containing two replicates of seven selected probes 448 for each of the 205 annotated genes of the R27 plasmid (NC_002305), as

449 previously described (Paytubi et al., 2014). The complete data set has been 450 deposited under accession number E-MTAB-4067 at

451 http:/www.ebi.ac.uk/arrayexpress.

\section{Acknowledgements}

453 This work was supported by grants from the Spanish Ministry of Science and 454 Innovation (CDS2008-0013, BFU2010-21836-C02-02, BIO2010-15417, 455 AGL2013-45339-R), the RecerCaixa program (2012/ACUP/00048) and the 456 Generalitat de Catalunya (2014SGR1260). 
457

458

459

460

461

462

463

464

465

466

467

468

469

470

471

472

473

474

475

476

477

478

479

480

481

482

483

484

485

486

487

488

489

490

491

492

\section{References}

Aberg, A., Shingler, V., and Balsalobre, C. (2008) Regulation of the fimB promoter: a case of differential regulation by ppGpp and DksA in vivo. Mol. Microbiol. 67: 1223-1241.

Alonso, G., Baptista, K., Ngo, T., and Taylor, D.E. (2005) Transcriptional organization of the temperature-sensitive transfer system from the IncHI1 plasmid R27. Microbiology 151: 3563-3573.

Baba, T., Ara, T., Hasegawa, M., Takai, Y., Okumura, Y., Baba, M., et al. (2006) Construction of Escherichia coli K-12 in-frame, single-gene knockout mutants: the Keio collection. Mol. Syst. Biol. 2: 2006.0008.

Battesti, A., Majdalani, N., and Gottesman, S. (2011) The RpoS-mediated general stress response in Escherichia coli. Annu. Rev. Microbiol. 65: 189213.

Bennett, P.M. (2008) Plasmid encoded antibiotic resistance: acquisition and transfer of antibiotic resistance genes in bacteria. Br. J. Pharmacol. 153 Suppl: S347-357.

Bremer, E., Gerlach, P., and Middendorf, A. (1988) Double negative and positive control of tsx expression in Escherichia coli. J. Bacteriol. 170: 108116.

Camacho, E.M., Serna, A., Madrid, C., Marqués, S., Fernández, R., de la Cruz, F., et al. (2005) Regulation of finP transcription by DNA adenine methylation in the virulence plasmid of Salmonella enterica. J. Bacteriol. 187: 5691-5699.

Dorman, C.J. (2014) H-NS-like nucleoid-associated proteins, mobile genetic elements and horizontal gene transfer in bacteria. Plasmid 75C: 1-11.

Doyle, M., Fookes, M., Ivens, A., Mangan, M.W., Wain, J., and Dorman, C.J. (2007) An H-NS-like stealth protein aids horizontal DNA transmission in bacteria. Science 315: 251-252.

Elton, T.C., Holland, S.J., Frost, L.S., and Hazes, B. (2005) F-like type IV secretion systems encode proteins with thioredoxin folds that are putative DsbC homologues. J. Bacteriol. 187: 8267-8277.

Forns, N., Baños, R.C., Balsalobre, C., Juárez, A., and Madrid, C. (2005) Temperature-dependent conjugative transfer of R27: role of chromosomeand plasmid-encoded Hha and H-NS proteins. J. Bacteriol. 187: 39503959.

Fox, R.E., Zhong, X., Krone, S.M., and Top, E.M. (2008) Spatial structure and 
nutrients promote invasion of IncP-1 plasmids in bacterial populations. ISME J. 2: 1024-1039.

Frost, L.S. and Koraimann, G. (2010) Regulation of bacterial conjugation: balancing opportunity with adversity. Future Microbiol. 5: 1057-1071.

Frost, L.S. and Manchak, J. (1998) F- phenocopies: characterization of expression of the $\mathrm{F}$ transfer region in stationary phase. Microbiology 144 ( Pt 9: 2579-2587.

Gibert, M., Juárez, A., Madrid, C., and Balsalobre, C. (2013a) New insights in the role of HtdA in the regulation of R27 conjugation. Plasmid 70: 61-68.

Gibert, M., Juárez, A., Zechner, E.L., Madrid, C., and Balsalobre, C. (2014) TrhR, TrhY and HtdA, a novel regulatory circuit that modulates conjugation of the IncHI plasmids. Mol. Microbiol. 94: 1146-1161.

Grindley, N.D., Grindley, J.N., and Anderson, E.S. (1972) R factor compatibility groups. Mol Gen Genet 119: 287-297.

Gruber, C.J., Lang, S., Rajendra, V.K.H., Nuk, M., Raffl, S., Schildbach, J.F., and Zechner, E.L. (2016) Conjugative DNA Transfer Is Enhanced by Plasmid R1 Partitioning Proteins. Front. Mol. Biosci. 3:32.

Gunton, J.E., Ussher, J.E.R., Rooker, M.M., Wetsch, N.M., Alonso, G., and Taylor, D.E. (2008) Entry exclusion in the IncHI1 plasmid R27 is mediated by EexA and EexB. Plasmid 59: 86-101.

Guyer, M.S., Reed, R.R., Steitz, J.A., and Low, K.B. (1981) Identification of a sex-factor-affinity site in E. coli as gamma delta. Cold Spring Harb. Symp. Quant. Biol. 45 Pt 1: 135-140.

Guynet, C., Cuevas, A., Moncalián, G., and de la Cruz, F. (2011) The stb operon balances the requirements for vegetative stability and conjugative transfer of plasmid R388. PLoS Genet. 7: e1002073.

Händel, N., Otte, S., Jonker, M., Brul, S., and ter Kuile, B.H. (2015) Factors that affect transfer of the Incl1 $\beta$-lactam resistance plasmid pESBL-283 between E. coli strains. PLoS One 10: e0123039.

Holt, K.E., Phan, M.D., Baker, S., Duy, P.T., Nga, T.V.T., Nair, S., et al. (2011) Emergence of a Globally Dominant IncHI1 Plasmid Type Associated with Multiple Drug Resistant Typhoid. PLoS Negl. Trop. Dis. 5: e1245.

Lawley, T.D., Gilmour, M.W., Gunton, J.E., Standeven, L.J., and Taylor, D.E. (2002) Functional and mutational analysis of conjugative transfer region 1 (Tra1) from the IncHI1 plasmid R27. J. Bacteriol. 184: 2173-2180.

Lawley, T.D., Gilmour, M.W., Gunton, J.E., Tracz, D.M., and Taylor, D.E. (2003) 
Functional and mutational analysis of conjugative transfer region 2 (Tra2) from the IncHI1 plasmid R27. J. Bacteriol. 185: 581-591.

Magnusson, L.U., Farewell, A., and Nyström, T. (2005) ppGpp: a global regulator in Escherichia coli. Trends Microbiol. 13: 236-242.

Maher, D. and Taylor, D.E. (1993) Host range and transfer efficiency of incompatibility group HI plasmids. Can. J. Microbiol. 39: 581-587.

Merkey, B. V, Lardon, L.A., Seoane, J.M., Kreft, J.-U., and Smets, B.F. (2011) Growth dependence of conjugation explains limited plasmid invasion in biofilms: an individual-based modelling study. Environ. Microbiol. 13: 24352452.

Miller, J.H. 1992. Short Course in Bact.Genet. Laboratory Manual. Cold Spring Harbor Laboratory Press.

Müller, C.M., Aberg, A., Straseviçiene, J., Emody, L., Uhlin, B.E., and Balsalobre, C. (2009) Type 1 fimbriae, a colonization factor of uropathogenic Escherichia coli, are controlled by the metabolic sensor CRP-CAMP. PLoS Pathog. 5: e1000303.

Paytubi, S., Aznar, S., Madrid, C., Balsalobre, C., Dillon, S.C., Dorman, C.J., and Juárez, A. (2014) A novel role for antibiotic resistance plasmids in facilitating Salmonella adaptation to non-host environments. Environ. Microbiol. 16: 950-962.

Paytubi, S., Aznar, S., Madrid, C., Balsalobre, C., Dillon, S.C., Dorman, C.J., and Juárez, A. (2013) A novel role for antibiotic resistance plasmids in facilitating Salmonella adaptation to non-host environments. Environ. Microbiol.16: 950-962.

Reisner, A., Molin, S., and Zechner, E.L. (2002) Recombinogenic engineering of conjugative plasmids with fluorescent marker cassettes. FEMS Microbiol. Ecol. 42: 251-259.

Rooker, M.M., Sherburne, C., Lawley, T.D., and Taylor, D.E. (1999) Characterization of the Tra2 Region of the IncHI1 Plasmid R27. Plasmid 41: 226-239.

Sambrook, J., Fritsch, E.F., and Maniatis, T. (1989) Molecular Cloning: A Laboratory Manual. Cold Spring Harbor Laboratory Press.

Schneider, C.A., Rasband, W.S., and Eliceiri, K.W. (2012) NIH Image to ImageJ: 25 years of image analysis. Nat. Methods 9: 671-675.

Shimada, T., Fujita, N., Yamamoto, K., and Ishihama, A. (2011) Novel Roles of cAMP Receptor Protein (CRP) in Regulation of Transport and Metabolism of Carbon Sources. PLoS One 6: e20081. 
566 Smillie, C., Garcillán-Barcia, M.P., Francia, M.V., Rocha, E.P.C., and de la Cruz, F. (2010) Mobility of plasmids. Microbiol. Mol. Biol. Rev. 74: 434-452.

Starcic, M., Zgur-Bertok, D., Jordi, B.J.A.M., Wösten, M.M.S.M., Gaastra, W., and van Putten, J.P.M. (2003) The cyclic AMP-cyclic AMP receptor protein complex regulates activity of the traJ promoter of the Escherichia coli conjugative plasmid pRK100. J. Bacteriol. 185: 1616-1623.

Taylor, D.E. and Levine, J.G. (1980) Studies of temperature-sensitive transfer and maintenance of $\mathrm{H}$ incompatibility group plasmids. J. Gen. Microbiol. 116: 475-484.

Will, W.R., Lu, J., and Frost, L.S. (2004) The role of H-NS in silencing F transfer gene expression during entry into stationary phase. Mol. Microbiol. 54: 769-782.

Zahrl, D., Wagner, M., Bischof, K., Bayer, M., Zavecz, B., Beranek, A., et al. (2005) Peptidoglycan degradation by specialized lytic transglycosylases associated with type III and type IV secretion systems. Microbiology 151: 3455-67.

Zheng, D., Constantinidou, C., Hobman, J.L., and Minchin, S.D. (2004) Identification of the CRP regulon using in vitro and in vivo transcriptional profiling. Nucleic Acids Res. 32: 5874-5893. 


\section{Figure legends.}

588 Figure 1. Effect of the physiological state of the cells in the conjugation

589 frequency of R27. A. Growth curve of strains AAG1 (R27) (black lines) and

590 MG1655 (grey lines) in PB medium. Arrows indicate the $\mathrm{OD}_{600}$ corresponding to

591 logarithmic $\left(\log , \mathrm{OD}_{600}\right.$ of 0.4$)$ and early-stationary (e-stat, $\mathrm{OD}_{600}$ of 2.0). A

592 representative experiment is shown. B. Conjugation rates were calculated using

593 cultures of both donor (AAG1(R27)) and recipient (MG1655) strains grown to

594 log or e-stat phase. The median of the conjugation frequency of three

595 experiments is shown.

596 Figure 2. Effect of the physiological state of the cells in the transcriptional

597 expression of the R27 genes. A. Fold change expression of the 205 ORFs

598 encoded in R27 plasmid between log and e-stat AAG1(R27) cells. The fold

599 change of +2 and -2 , indicating significantly higher expressed in log and e-stat

600 cultures respectively, is labelled with dashed lines. Expressed genes were

601 classified as tra genes (black bars) and non tra genes (grey bars). Genes with

602 signal values lower than 100 fluorescence units in both culture conditions were

603 arbitrarily considered as non-expressed (white bars). B. Transcriptional

604 expression of the different tra genes in log (grey bars) and e-stat (black bars)

605 cultures is shown as arbitrary units (fluorescence intensity). C. Transcription

606 analysis by semi-quantitative RT-PCR of seven selected genes, representative

607 of the six tra operons from the AAG1(R27) strain grown as in A up to either log

608 or e-stat phase. 16S rRNA was used as a control.

609 Figure 3. Growth phase regulation of R27 conjugation in different genetic

610 backgrounds. A. Frequency of conjugation using as donor cells AAG1(R27) and

611 derivative strains grown to either log or e-stat phase. The median of the 
612 conjugation frequency of three experiments is shown. B. RT-PCR analyses of 613 trhA expression in WT, htdA and trhR strains. The amount of total RNA used in 614 each case is shown. Relative levels of cDNA are indicated below each panel.

615 Figure 4. Ectopic expression of HtdA and TrhR/TrhY vanish the growth phase 616 dependency of R27 conjugation. Conjugation frequencies of the AAG1(R27) 617 strain carrying the indicated plasmids. Donor cultures were grown to either log or e-stat phase in the presence of glucose or arabinose. The median of the conjugation frequency of three experiments is shown.

Figure 5. cAMP modulates conjugation in an HtdA-dependent manner during logarithmic phase of growth. A. RT-PCR analyses of trhA in log cultures of WT and cya strains. 16S rRNA was used as a control. B. Frequency of conjugation using as donor log or e-stat AAG1 cells and its cya counterpart carrying either $\mathrm{R} 27\left(h t d A^{+}\right)$or $\operatorname{drR} 27\left(h t d A^{-}\right)$. The median of the conjugation frequency of three experiments is shown. C. Frequency of conjugation using as donor AAG1 cells

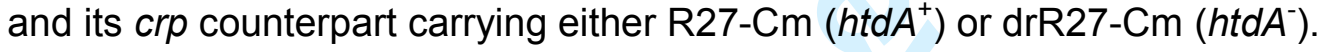
Cultures of the donor cells were grown until log phase. The median of the conjugation frequency of three experiments is shown. D. Transcriptional expression of $h t d A$ using a chromosomal lacZ fusion with the promoter of the AN operon (AAG1-AN strain). $\beta$-galactosidase activity (Miller units) was determined in cultures of strain AAG1-AN, and its cya and crp derivatives in the absence or presence of the R27 plasmid grown as in A. E. htdA transcriptional expression in cultures of the WT (AAG1-AN) and its cya derivative strain in the presence or the absence of cAMP. Bacterial cultures were grown in LB at $25^{\circ}$ to an $\mathrm{OD}_{600 \mathrm{~nm}}$ of 0.1 , cAMP $(5 \mathrm{mM})$ was added and $\beta$-galactosidase activity (Miller units) was determined after 2 hours incubation. 
637 Figure 6. Proposed model of regulation of R27 plasmid conjugation in 638 logarithmic (A) or stationary (B) phase of growth.

639 
Table 1. Bacterial strains and plasmids used in this work

\begin{tabular}{|c|c|c|}
\hline Strain & Description & Source of reference \\
\hline MG1655 & $\mathrm{F}-$, ilvG, rph1 & (Guyer et al., 1981) \\
\hline AAG1 & MG1655 $\Delta / a c Z$ & (Aberg et al., 2008) \\
\hline JFV2 & AAG1 $\Delta r e l A \Delta s p o T$ & (Aberg et al., 2008) \\
\hline JW5437 & BW25113 rpoS:::Km ${ }^{R}$ & (Baba et al., 2006) \\
\hline AAG1 rpos & AAG1 rpoS::Km $\mathrm{Km}^{\mathrm{R}}$ & This work \\
\hline AAG1 hns & AAG1 hns:::Km ${ }^{R}$ & (Gibert et al., 2014) \\
\hline AAG1 cya & AAG1 cya::Tmp ${ }^{R}$ & $\begin{array}{l}\text { (Gibert et al., unpublished } \\
\text { results) }\end{array}$ \\
\hline AAG1-AN & AAG1 $P_{A N}:: / a c Z-K m^{R}$ in attB site & (Gibert et al., 2013) \\
\hline AAG1-AN cya & AAG1-AN cya::Tmp ${ }^{R}$ & This work \\
\hline BRE2055 & $\Delta($ crp)96 zhd- 732::Tn10 & (Bremer et al., 1988) \\
\hline AAG1 crp & AAG1 $\Delta(c r p) 96$ zhd- 732::Tn10 & This work \\
\hline AAG1-AN crp & $\begin{array}{l}\text { AAG1-AN } \Delta(c r p) 96 \text { zhd- } \\
732:: \operatorname{Tn} 10\end{array}$ & This work \\
\hline Plasmid & & \\
\hline $\mathrm{R} 27$ & IncHI1 Tc ${ }^{\mathrm{R}}$ & (Grindley et al., 1972) \\
\hline drR27 & R27 htdA::IS10 & (Gibert et al., 2013) \\
\hline $\mathrm{R} 27-\mathrm{Cm}$ & $\begin{array}{l}\text { insertion of cat gene from } \\
\text { pAR92 into tetA gene of } R 27 \text {, } \\
\mathrm{Cm}^{\mathrm{R}}\end{array}$ & $\begin{array}{l}\text { (Gibert et al., unpublished } \\
\text { results) }\end{array}$ \\
\hline drR27-Cm & $\begin{array}{l}\text { insertion of cat gene from } \\
\text { pAR92 into tetA gene of } \mathrm{drR} 27 \text {, } \\
\mathrm{Cm}^{\mathrm{R}}\end{array}$ & $\begin{array}{l}\text { (Gibert et al., unpublished } \\
\text { results) }\end{array}$ \\
\hline R27 hns & $\mathrm{R} 27$ hns::Cm $\mathrm{Cm}^{\mathrm{R}}$ & (Gibert et al., 2014) \\
\hline R27trhR-Tmp & R27 trhR::Tmp ${ }^{R}$ & (Gibert et al., 2014) \\
\hline pBADtrhRY & pBAD18 + trhRtrhY & (Gibert et al., 2014) \\
\hline pBADhtdA & $\mathrm{pBAD} / \mathrm{HisB}+h t d A$ & (Gibert et al., 2014) \\
\hline pAR92 & $\begin{array}{l}\text { cat- } \mathrm{P}_{\mathrm{A} 1 / 04 / 03^{-}}-c f p^{*}-\mathrm{T}_{0} \text { cassette, } \\
\mathrm{Amp}^{\mathrm{R}}, \mathrm{Cm}^{\mathrm{R}}\end{array}$ & (Reisner et al., 2002) \\
\hline
\end{tabular}


A

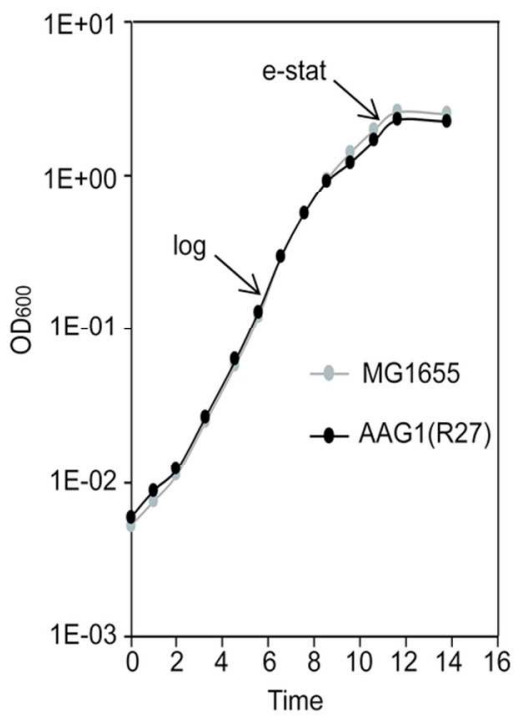

B

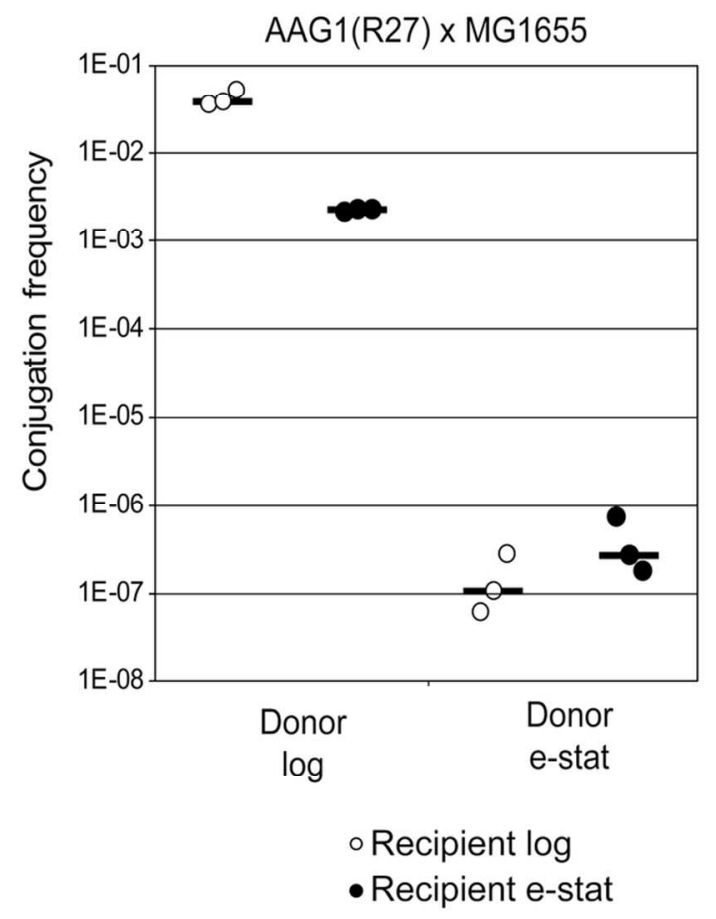

Figure 1

Figure 1

$96 \times 70 \mathrm{~mm}(300 \times 300 \mathrm{DPI})$ 

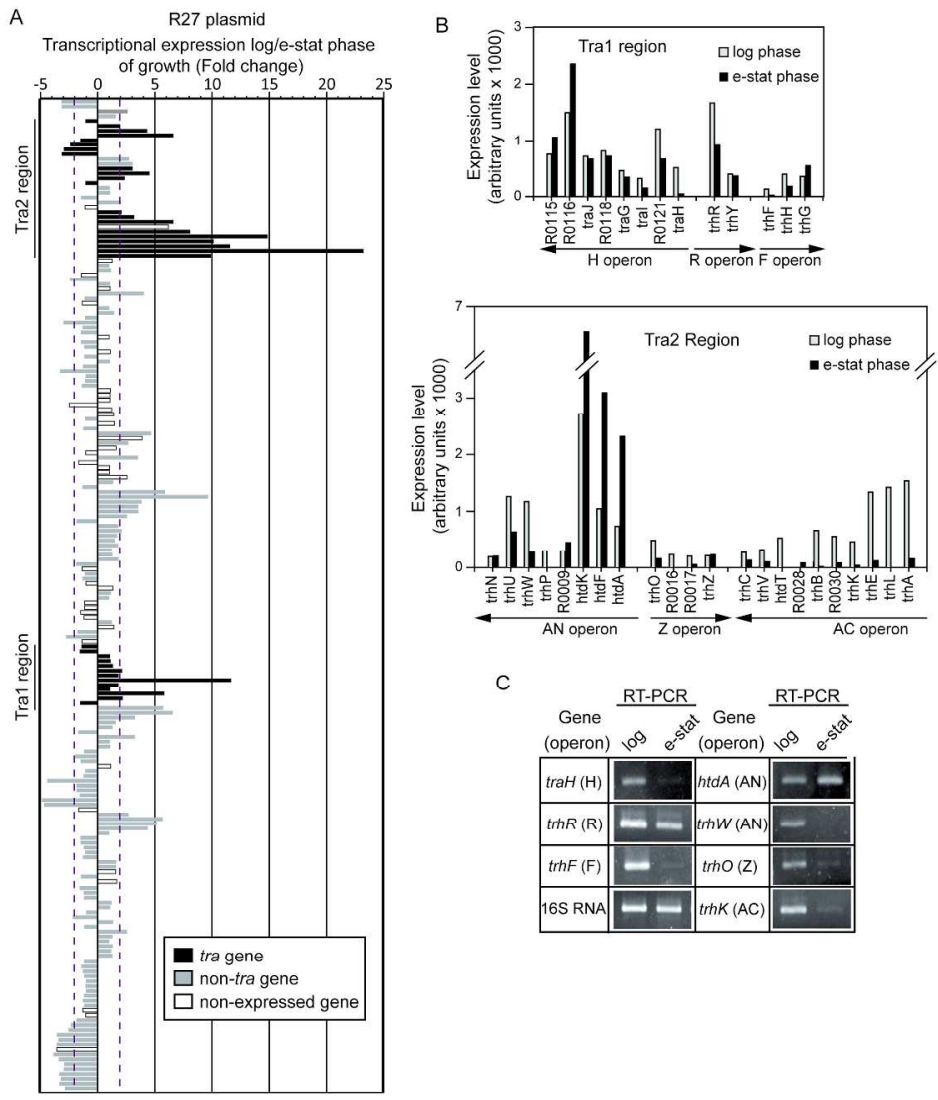

c

\begin{tabular}{|c|c|c|c|}
\hline \multicolumn{3}{|c|}{ RT-PCR } & RT-PCR \\
\hline $\begin{array}{r}\text { Gene } \\
\text { (operon }\end{array}$ & $0^{9} e^{5}$ & $\begin{array}{c}\text { Gene } \\
\text { (operon) }\end{array}$ & $0^{9} e^{-5}$ \\
\hline $\operatorname{traH}(\mathrm{H})$ & - & htdA (AN) & -6 \\
\hline $\operatorname{trh} \kappa(\mathrm{R})$ & -5 & $t h t W(\mathrm{AN})$ & - \\
\hline $\operatorname{trhF}(F)$ & - & $\operatorname{trhO}(\mathrm{Z})$ & 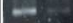 \\
\hline $16 \mathrm{~S}$ RNA & -- & $\operatorname{trm} K(\mathrm{AC})$ & - \\
\hline
\end{tabular}

Figure 2

Figure 2

$246 \times 232 \mathrm{~mm}(300 \times 300 \mathrm{DPI})$ 


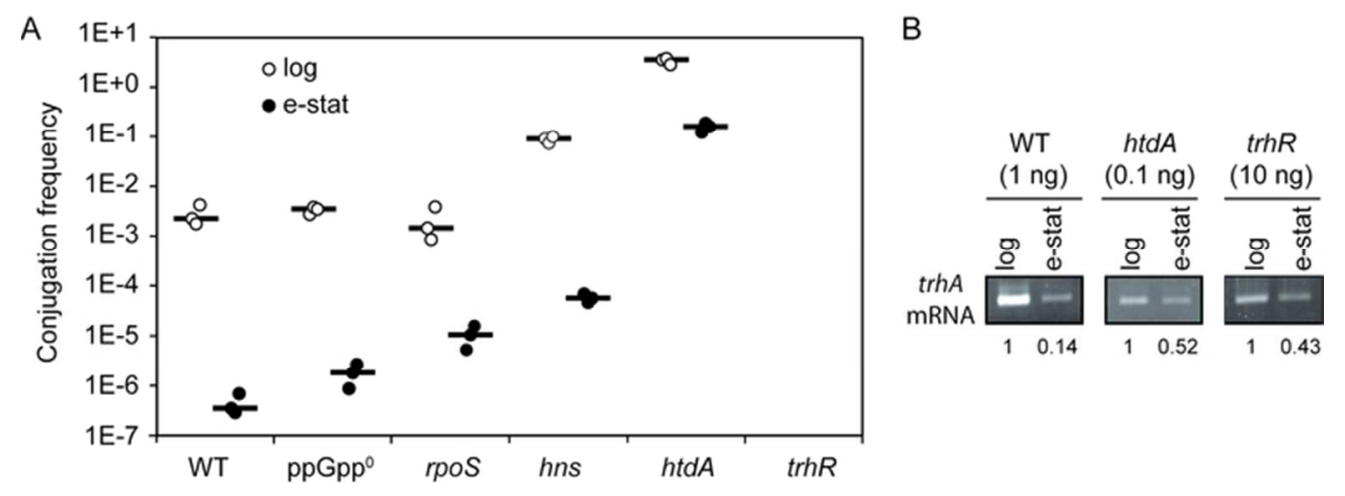

Figure 3

Figure 3

$63 \times 23 \mathrm{~mm}(300 \times 300 \mathrm{DPI})$ 


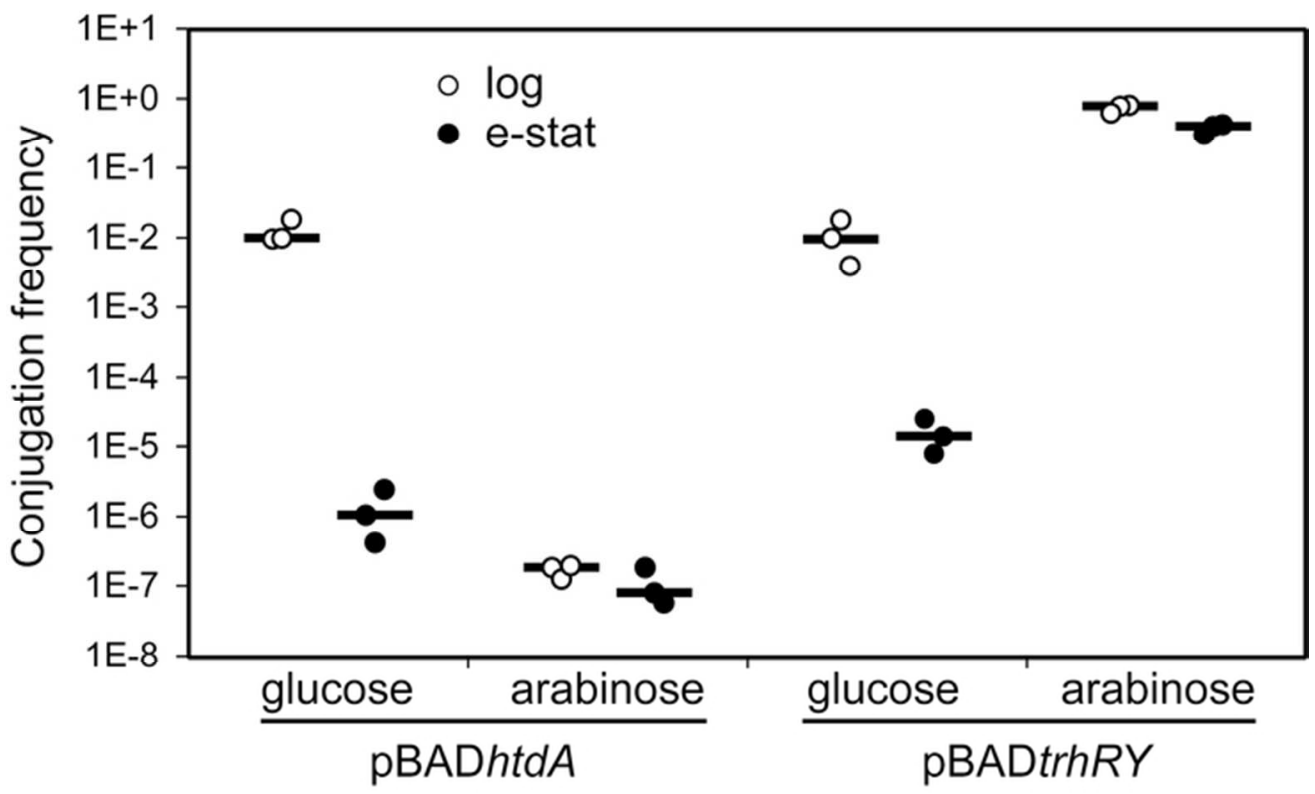

Figure 4

Figure 4

$62 \times 37 \mathrm{~mm}(300 \times 300$ DPI $)$ 

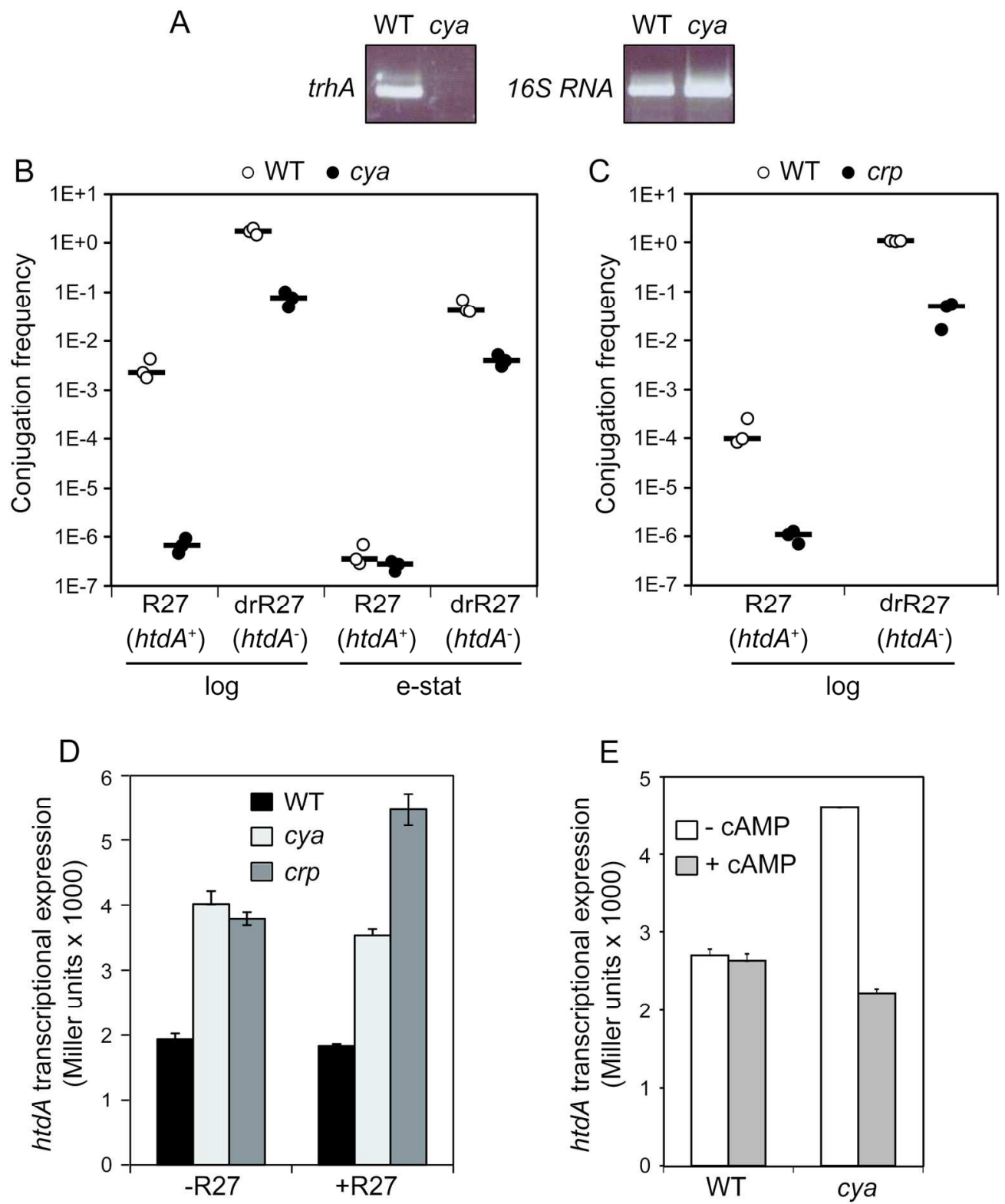

Figure 5

Figure 5

$162 \times 195 \mathrm{~mm}(300 \times 300$ DPI $)$ 


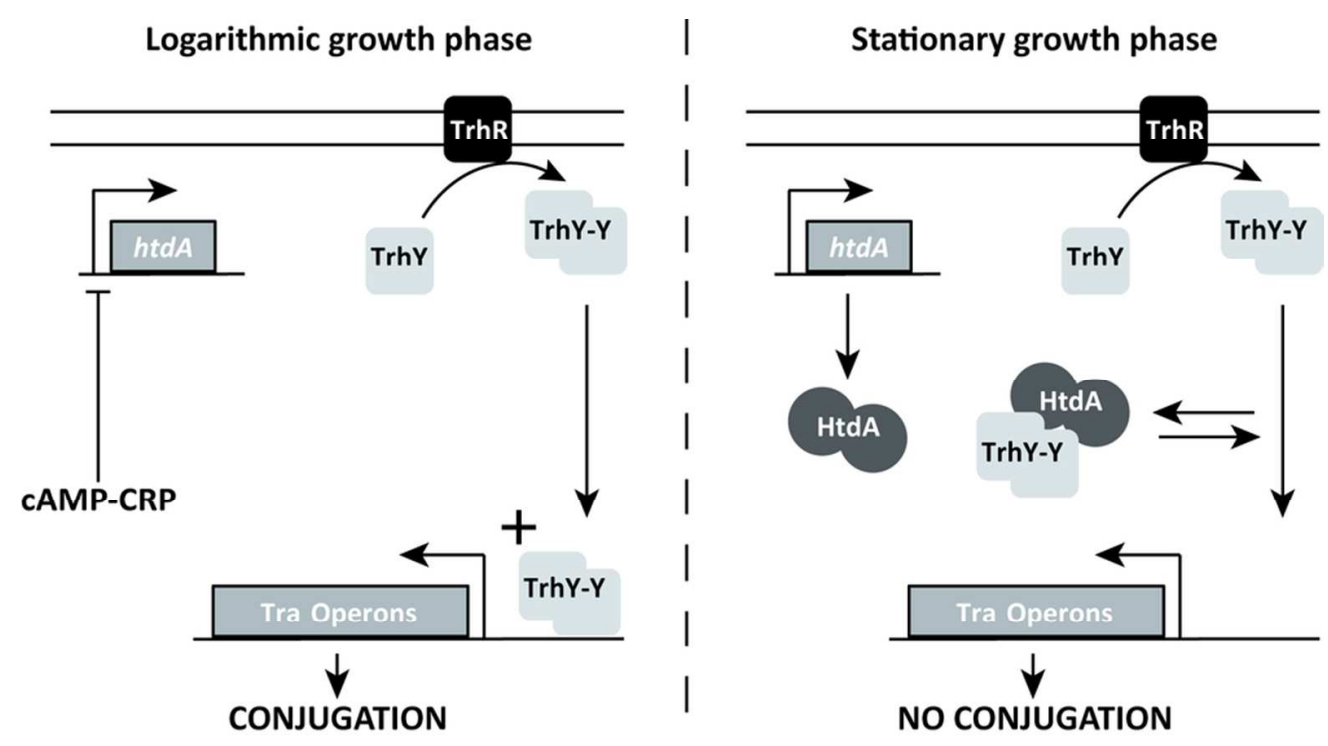

Figure 6

Figure 6

$85 \times 47 \mathrm{~mm}(300 \times 300 \mathrm{DPI})$ 